\title{
Development of Computer Vision System to Extract Kansei Information from Facial Expressions
}

\author{
Yutaka Sasaki*1), Mayu Emmi²) and Yasuyuki Takahashi' ${ }^{1)}$ \\ 1) Faculty of Regional Environment Science, Tokyo University of Agriculture, 1-1-1 Sakuragaoka, Setagaya, Tokyo 156-8502, Japan \\ 2) Graduate School of Agriculture, Tokyo University of Agriculture, 1-1-1 Sakuragaoka, Setagaya, Tokyo 156-8502, Japan
}

\begin{abstract}
In the Japanese language, the word "Kansei" is used to indicate mental states or activities that occur in response to stimulation from the outside world. For example, emotion is defined as a component of Kansei. In the West, words such as sensibility and perceptiveness are often used with meanings similar to Kansei. Furthermore, in the Japanese field of Kansei engineering, designers strive to integrate human feelings and sensibilities more closely with manufacturing and production activities. Accordingly, if Kansei-related information can be extracted in various situations, it is believed that distinctive design, development, and evaluation processes become possible. In this paper, we describe the fabrication of an inexpensive and near real-time system capable of extracting Kansei information from user facial expressions. To accomplish this, we developed a computer vision system using the free OpenCV image-processing library. The characteristic values calculated from these inputs were the variation (the normalized polygon area change ratio) of four items: the inner end of the eyebrows, the upper and lower parts of the eyes, and the corners of the mouth, which were then calculated using seven face nodes. Three Kansei information types were targeted: positive Kansei information (appreciation, fun, and happiness), negative Kansei information (unpleasantness and distaste), and Kansei information associated with shock or surprise. With the assistance of 12 test subjects, we began working to obtain specific Kansei knowledge, and quickly determined that the relationships between facial expressions and Kansei information were highly individual in nature. Therefore, in the next stage, we formulated an algorithm to discriminate the three Kansei information types while focusing on a single subject, and obtained good results. However, since facial reactions vary significantly between individual human beings, it is currently necessary to prepare a seven-point template image in advance for each person to be examined, and then to adjust the algorithm parameters after measuring their facial expressions in order to obtain Kansei information. Despite these initial difficulties, our results show that an inexpensive and near real-time computer vision system capable of extracting facial expression Kansei information can be constructed. We believe that such systems can make it possible to apply Kansei information decision support to a variety of automatic evaluation and design processes.
\end{abstract}

\section{Keywords}

Kansei, computer vision, automatic evaluation and design, decision support system, Open CV

\section{Introduction}

In the Japanese language, the word "Kansei" is used to indicate mental states or activities that occur in response to stimulation from the outside world. For example, emotion is defined as a component of Kansei. In the West, words such as sensibility and perceptiveness are used with meanings similar to Kansei.

* Corresponding Author

E-mail: y3sasaki@nodai.ac.jp
In this study, we aim to apply Kansei engineering, which is a field in which Kansei information and human imagery are adapted to physical design elements in order to create products, environments, food, and services that are compatible with Kansei. By utilizing Kansei information, we believe that the following will become possible:

- Design, development and evaluation of new agricultural products and foods with colors, shapes, scents, etc., that evoke strong consumer approval;

- Development of unique marketing methods; design and 
evaluation of office and living spaces that incorporate green amenities (Asaumi et al. 1995);

- Landscape creation and evaluation for cities, farming villages and gardens, with comfort and healing effects;

- Development of agricultural machines with an appearance, or level of comfort, that evokes strong consumer approval;

- Development of agri-robots capable of Kansei communication (Sasaki 2011);

- Welfare and nursing support using animals, plants and/or artificial life.

In this study, two specific application examples are considered (Fig. 1). The first example relates to the disaster reconstruction program in Fukushima Prefecture and corresponding efforts by the Tokyo University of Agriculture to create a Cyber field simulation system that can be used for restoration of farming and mountain villages in disaster areas. This effort consists of building a base simulation system by adding geographic information system (GIS) map information and three-dimensional (3D) space information obtained using a mobile mapping system. It was designed for the restoration of farming and mountain villages, and can be used to construct a regional model simulator that gives consideration to disaster prevention and post-reconstruction landscaping. If Kansei information can be utilized, this can be expected to lead to the creation of a landscape simulator. In other words, if Kansei information proves to be useful, it may be possible to automatically design landscapes that "heal" inhabitants, or make them feel more positive, when redeveloping rural settlements after earthquakes or other disasters. Mobile mapping is the process of collecting geospatial data from vehicles equipped with remote sensing systems. The second example in the present paper is the development of an educational application related to food and the environment for tablet personal computers (PCs). Until recently, the effects of a variety of education programs and efforts could only be evaluated through questionnaire surveys and similar methods. However, when Kansei information such as the degree to which students feel interested (e.g., whether they feel bored or stimulated) can be obtained, it can be used for the evaluation of educational applications and software, and other education related improvements.

As described above, this section of the study focuses, in particular, on the fabrication of an inexpensive and near real-time system to extract Kansei information from the facial expressions of a test subject via computer vision, and the related obtained Kansei knowledge, which was then used to create a discriminatory algorithm for discerning facial expressions.

The present study has the following goals:

1. Determine the relationship between facial expression and Kansei information for multiple subjects;

2. Develop a computer vision system capable of near realtime identification of Kansei information based facial expressions.

It is said that $55 \%$ of all emotional indicators, which are a part of Kansei information, can be presented by facial expressions (Mehirabian 1986). Accordingly, in the present study, we focus on facial expression information because of its importance in presenting emotional context, and because a facial expression recognition system can be easily achieved using a camera and image processing software, without requiring special sensors. Additionally, facial expressions can be measured without contact and can be easily used as feedback information. By developing the system further, we believe that Kansei information at the unconscious level, which cannot be captured through questionnaire surveys, can also be obtained.

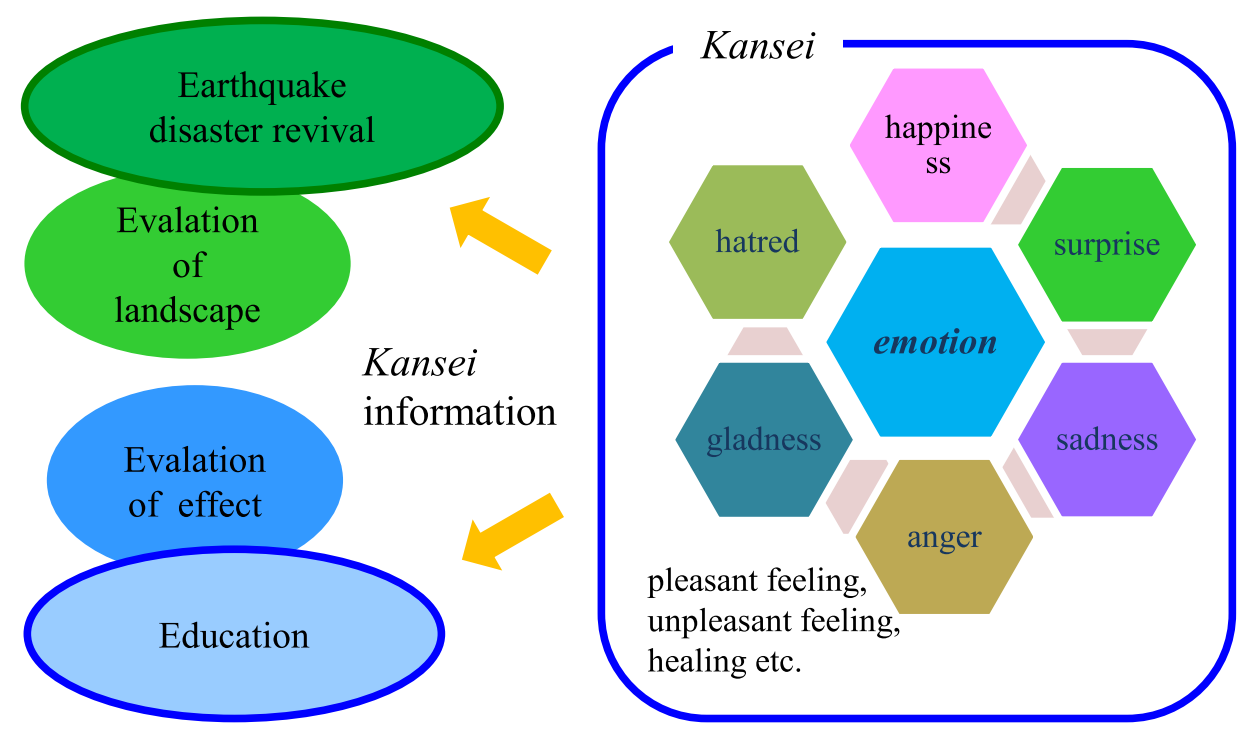

Fig. 1 Image of Kansei application 
In our earlier studies (Sasaki et al. 2007, 2009), nodes were placed on the faces of test subjects and their movements were tracked. However, that system required physical contact and was expensive. Another related study extracted Kansei information from facial expressions (Zisheng et al. 2010), but it could not operate in real time. In fact, a number of studies aimed at more fully grasping Kansei information have been carried out (e.g., Fujimura 2008).

However, no inexpensive camera-based computer vision system for obtaining near real-time Kansei information has been developed to date. Therefore, if such a system could be developed, it would be superior in terms of speed and cost effectiveness, and could be applied in food and environmental fields.

\section{Development System and Kansei Extraction Method}

Based on the results of our earlier study (Sasaki et al. 2007) and other related studies, it is known that people tend to express Kansei information using their eyes and mouth. Based on the facial graph created by Ayinde and Yang (2002), in order to obtain Kansei information, a total of seven facial nodes must be used. These are the inner ends of the eyebrows, the inner corners of the eyes, the outer corners of the eyes, the upper eyelids, the lower eyelids, and both corners of the mouth. Fig. 2 shows these seven facial nodes on the developed computer vision system. The node positions are determined using a template matching technique that searches for a partial image signal (target) in the image that corresponds to a known template signal. The position of an object is detected by first storing a region of the object to be traced in each image of a video as a template image, and then detecting a region that has a high degree of similarity to the template image in a time-series manner. The template used was a $19 \times 19$ pixel image. The seven facial nodes were then connected, which permitted the creation of four polygons corresponding to the inner end of the eyebrows (three facial nodes: inner end of the eyebrow, inner corner of the eyes, and outer corner of the eyes), the upper (three facial nodes: inner corner of the eyes, outer corner of the eyes, and upper eyelids) and lower parts of the eyes (three facial nodes: inner corner of the eyes, outer corner of the eyes, and lower eyelids), and the corners of the mouth (three facial nodes: inner corner of the eyes and both corners of the mouth), as shown in Fig. 3. These seven points were chosen because Kansei information is readily visible in the eyes and mouth of human subjects, and thus allows us to raise the processing speed. In this study, we used the Windows 7 operating system, the free OpenCV image-processing library, and Visual Studio 2010 for software development. We used OpenCV 2.3.1 (iostream library) for camera image acquisition and identification of the pattern matching characteristics/amounts. Using a common PC (Vostro 3550, Dell Inc., Intel Core i3 2350M, memory size: 4 GB, ATI HD Switchable Graphics) with a built-in Web camera, $640 \times 480$ pixel images were acquired. The distance between the camera and the subject was generally $50 \mathrm{~cm}$; it was found that facial nodes could be correctly traced for distances of 20 to $60 \mathrm{~cm}$. In order to increase the probability of proper facial node recognition, subjects did not wear glasses in this study.

The polygon areas were then calculated and extraction of Kansei information was attempted. In this paper, $N(i)$ is defined as a facial node and the following definitions are used: $N(1)$ indicates the inner end of the eyebrow, $N(2)$ indicates the upper eyelid, $N(3)$ indicates the lower eyelid, $N(4)$ indicates the inner corner of the eye, $N(5)$ indicates the outer corner of the eye, $N(6)$ indicates the left corner of the mouth, and $N(7)$ indicates the right corner of the mouth. The parameters used for facial expression analysis were the normalized polygon area change

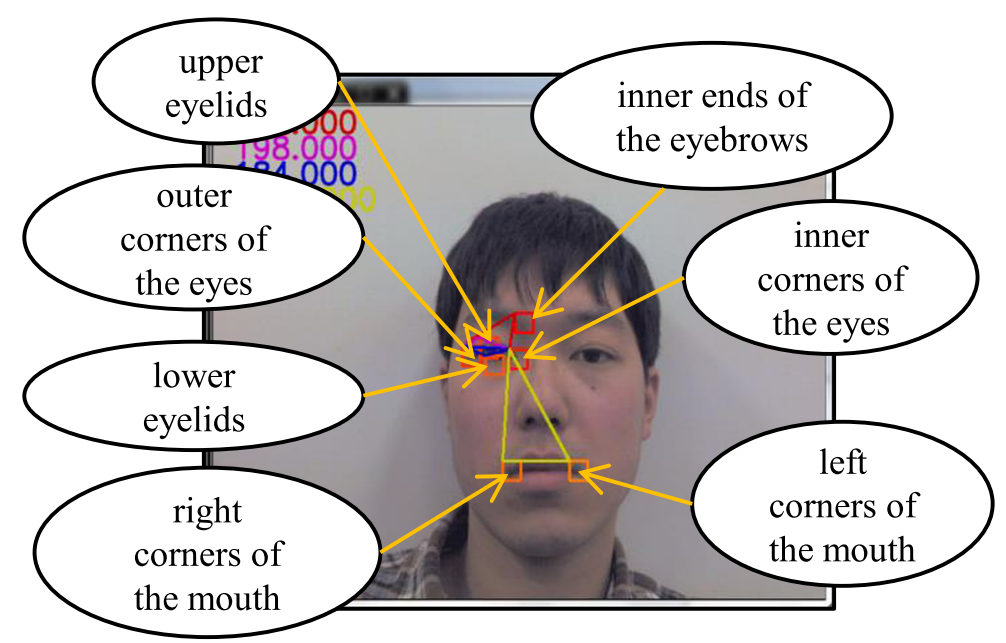

Fig. 27 face nodes for polygon areas 
ratio $\Delta A(i, j, k)$, as calculated using the following equation:

$$
\Delta A(i, j, k)=100 \cdot \frac{A_{t k}(i, j, k) / s c a l e^{2}-A_{t 0}(i, j, k)}{A_{t 0}(i, j, k)}
$$

where

$$
\text { scale }=L_{t k}(4,5) / L_{t 0}(4,5),
$$

which allows efficient evaluation of the back and forth head movements of each subject in the direction towards the camera (zoom in/out), as follows:

$$
\begin{aligned}
A_{t}(i, j, k)= & \frac{1}{2} \cdot \mid\left\{x_{t}(i) \cdot y_{t}(j)-x_{t}(j) \cdot y_{t}(i)\right\} \\
& +\left\{x_{t}(j) \cdot y_{t}(k)-x_{t}(k) \cdot y_{t}(j)\right\} \\
& +\left\{x_{t}(k) \cdot y_{t}(i)-x_{t}(i) \cdot y_{t}(k)\right\} \mid
\end{aligned}
$$

Hereafter, $\Delta A$ is defined as the normalized polygon area change ratio, $t$ is the time ( $t k$ : time of Kansei facial expression, to: time of normal facial expression), $i, j$, and $k$ are node numbers, $x_{t}(i)$ and $y_{t}(i)$ are the $x$ and $y$ coordinates, respectively, of $N(i)$ at time $t, L_{t}(i, j)$ is the distance between $N(i)$ and $N(j)$ at time $t$, scale indicates the scale ratio for normalization at times $t k$ and $t 0$, and $A_{t}(i, j, k)$ is the area of the polygon (triangle) formed by $N(i)-N(j)-N(k)$ at time $t$. The $\Delta A$ values were calculated for the four polygons at the inner ends of the eyebrows, upper parts of the eyes, lower parts of the eyes, and corners of the mouth. Since one of the goals of this study was to evaluate the possibility of using Kansei information to create pleasing products and environments, we focused on facial expressions associated with positive Kansei information (fondness, enjoyment, happiness, and pleasure), negative Kansei information (distaste, sadness, and unpleasantness), and surprise Kansei information.

The following experimental method was used. After the purpose of the experiments, the experimental procedure and the precautions taken, were explained to the test subjects, they were asked to provide written informed consent and give their ages and genders. They were then given an overview of the contents of the target Kansei expressions and instructed to practice those expressions using a mirror, after which the experiments to obtain Kansei expression information were conducted. Although the test subjects made their Kansei expressions intentionally, they were asked to make them as naturally as possible. The test subjects were 12 men (ages: 18-22). For these 12 persons, 50 data images were acquired for each normal, positive, negative, and surprise Kansei expression (2,400 images in total). $\Delta A$ values were then computed from the image data node changes for each Kansei expression using the mean node locations for 50 normal images. There were only slight variations between the 50 normal images.

\section{Results and Discussion}

\section{Knowledge about Kansei obtained from multiple persons}

After confirming that changes in Kansei information could be recognized by our current system in the preliminary experiments, we then used the system to obtain this information from each of the 12 test subjects. Fig. 4 shows the obtained positive, negative, and surprise Kansei information, as well as an example of the program execution results, and Fig. 5 shows the data for the 12 subjects. In Fig. 5, the x-axis shows the subject number and the y-axis shows the change rate. In addition, Fig. 5(a) shows positive Kansei information, Fig. 5(b) shows negative Kansei information, and Fig. 5(c) shows surprise Kansei information. From the results of the four polygons created using
(1) inner end of the eyebrow
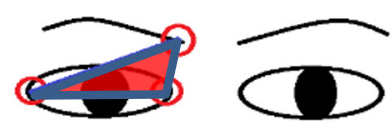

(3) lower part of the eye

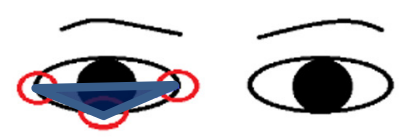

(2) upper part of the eye

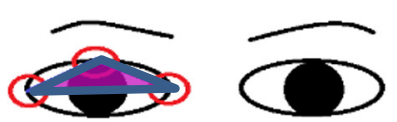

(4) corners of the mouth

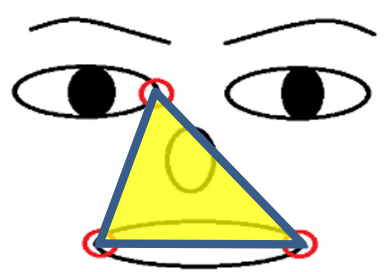

Fig. 34 polygons for Kansei extraction 
rectangles to connect the face nodes, and by connecting the polygons with lines, changes in the absolute values of $\Delta A$ were determined, and are listed in Table 1 . We found that the direction of the four polygons accompanying an expression alteration varied between the individual test subjects. In order to consider which areas should be focused on, based on their size and direction, the absolute variations were calculated (Table 1). Moreover, in order to investigate what trends accompany direction variations when multiple persons are considered, the poly-

Table 1 Absolute value changes of each polygon area* (\%)

\begin{tabular}{lccc}
\hline & $\begin{array}{c}\text { positive } \\
\text { Kansei }\end{array}$ & $\begin{array}{c}\text { negative } \\
\text { Kansei }\end{array}$ & $\begin{array}{c}\text { surprise } \\
\text { Kansei }\end{array}$ \\
\hline Inner end of the eyebrows & $15.3 \pm 11.7$ & $12.9 \pm 7.8$ & $14.6 \pm 11.8$ \\
Upper part of the eyes & $18.4 \pm 13.9$ & $13.7 \pm 9.9$ & $15.1 \pm 9.9$ \\
Lower part of the eyes & $18.4 \pm 19.3$ & $15.7 \pm 10.4$ & $14.6 \pm 12.3$ \\
Corners of the mouth & $28.8 \pm 15.7$ & $11.8 \pm 5.7$ & $17.9 \pm 12.8$ \\
\hline
\end{tabular}

$*$ average \pm standard deviation

Table 2 Subject percentage about the change direction (positive Kansei)

\begin{tabular}{lcc}
\hline & Plus (\%) & Minus (\%) \\
\hline Inner end of the eyebrows & 50.0 & 50.0 \\
Upper part of the eyes & 41.7 & 58.3 \\
Lower part of the eyes & 25.0 & 75.0 \\
Corners of the mouth & 83.3 & 16.7 \\
\hline
\end{tabular}

Table 3 Subject percentage about the change direction (negative Kansei)

\begin{tabular}{lcc}
\hline & Plus (\%) & Minus (\%) \\
\hline Inner end of the eyebrows & 16.7 & 83.3 \\
Upper part of the eyes & 25.0 & 75.0 \\
Lower part of the eyes & 8.3 & 91.7 \\
Corners of the mouth & 33.3 & 66.7
\end{tabular}

Table 4 Subject percentage about the change direction (surprise Kansei)

\begin{tabular}{lcc}
\hline & Plus (\%) & Minus (\%) \\
\hline Inner end of the eyebrows & 91.7 & 8.3 \\
Upper part of the eyes & 58.3 & 41.7 \\
Lower part of the eyes & 58.3 & 41.7 \\
Corners of the mouth & 75.0 & 25.0 \\
\hline
\end{tabular}

gon variation percentages were summarized, as shown in Tables 2-4.

As can be seen in Table 1, the results confirm that changes can be identified, and that the constructed system can be useful. Information obtained from multiple persons is next examined. For positive Kansei information, the changes in $\Delta A$ were

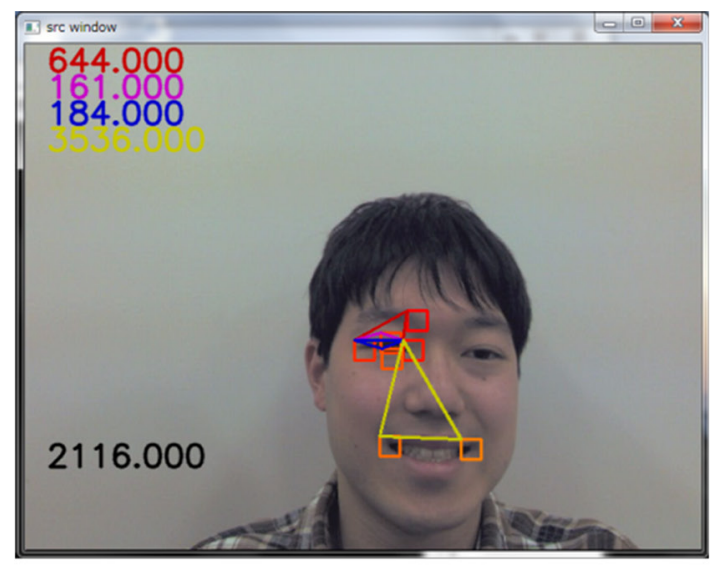

\section{(a) positive Kansei}

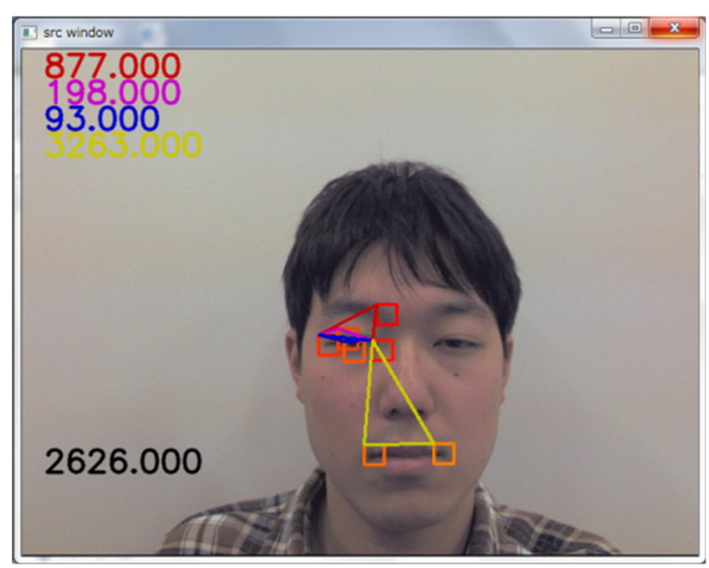

\section{(b) negative Kansei}

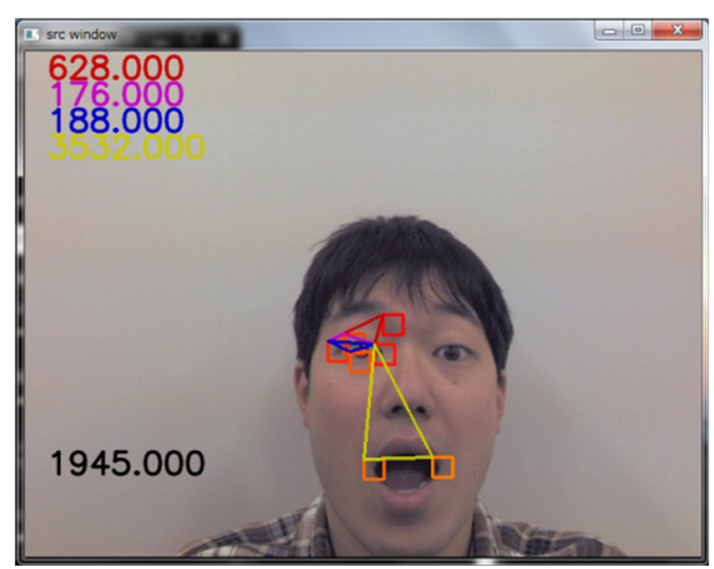

(c) surprise Kansei.

Fig. 4 Examples of the acquired images 


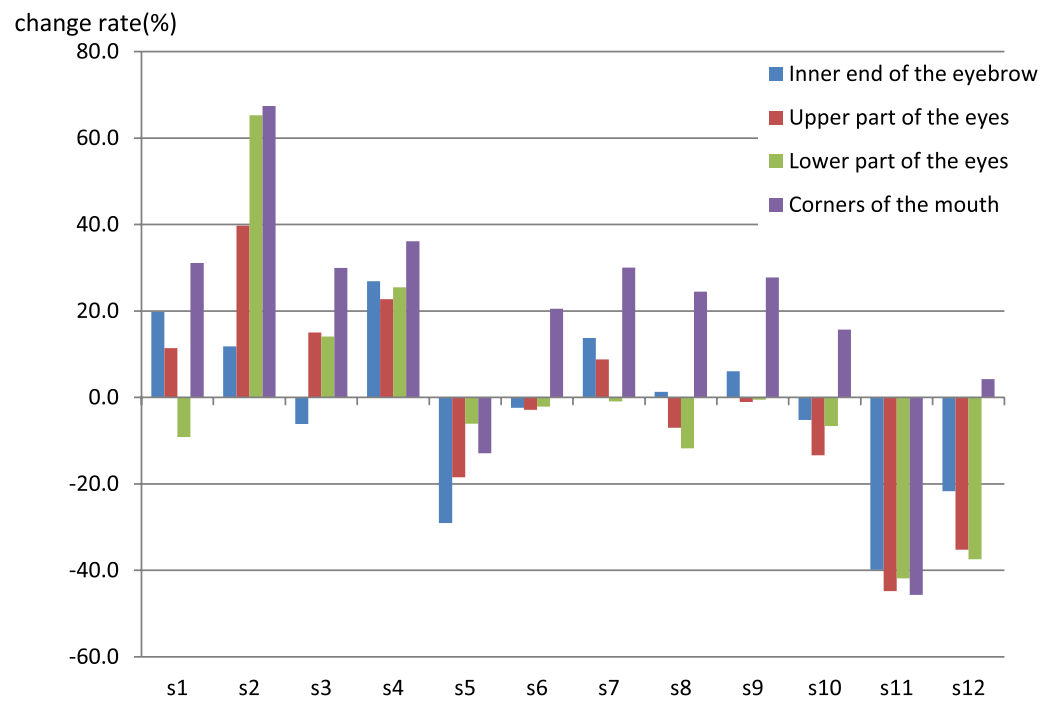

(a) positive Kansei

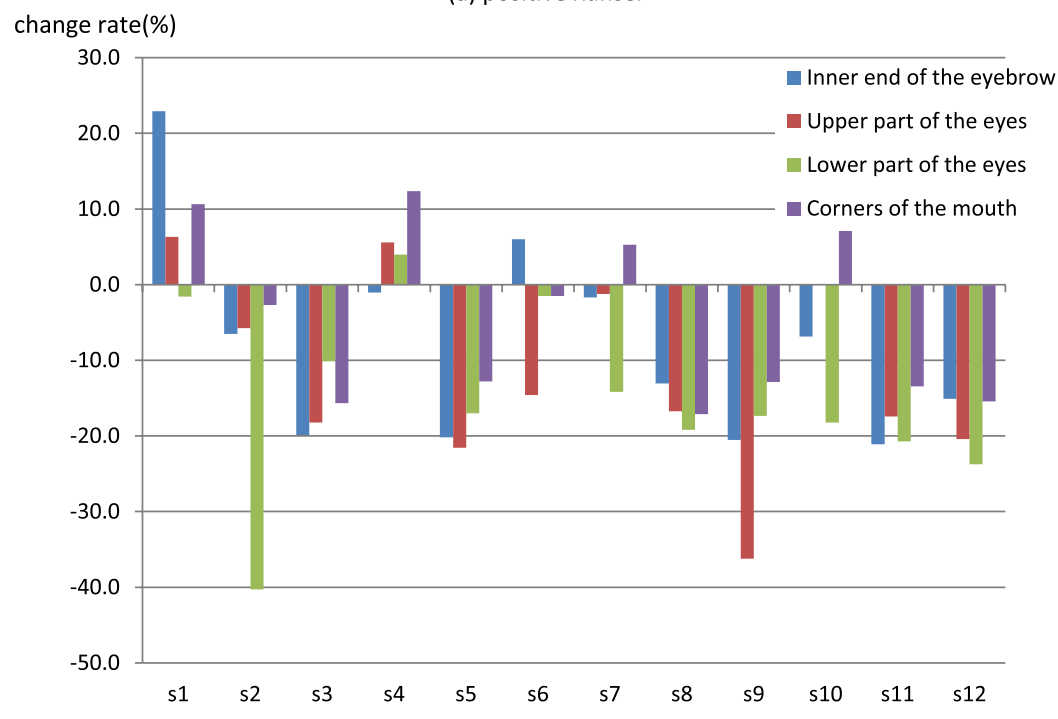

(b) negative Kansei

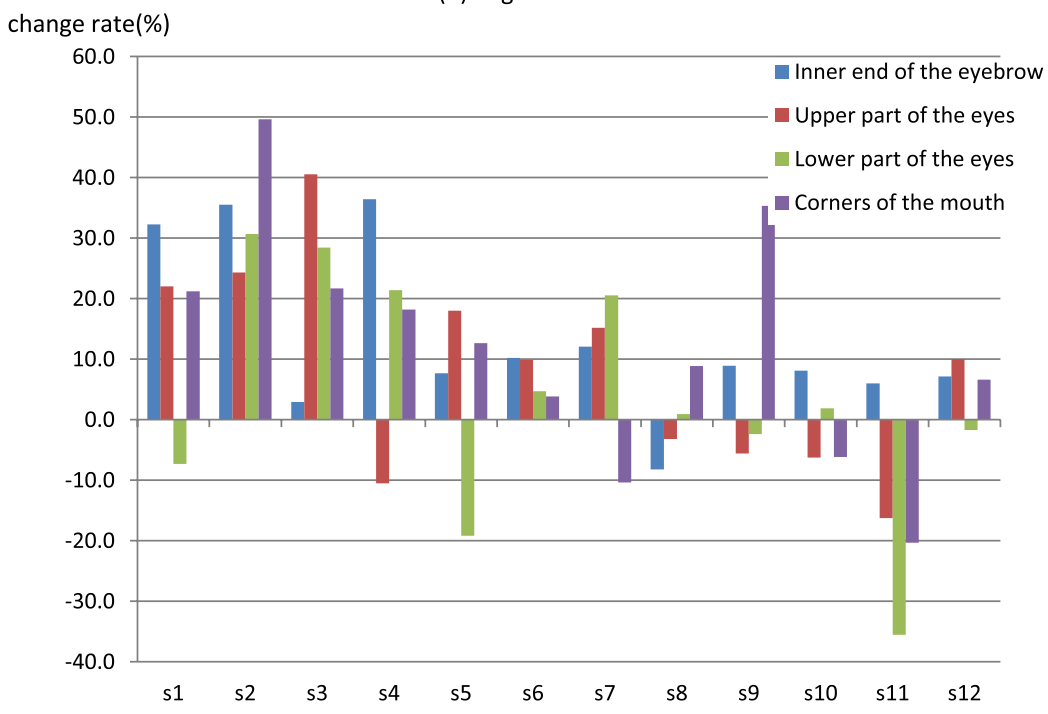

(c) surprise Kansei

Fig. 512 subjects data about change rate of polygon area 
approximately $15-29 \%$, and the changes at the corners of the mouth were about $29 \%$, which was notably higher than the others. For negative Kansei information, the changes were about $11-16 \%$, with the change at the lower parts of the eye (approximately $16 \%$ ) found to be slightly higher than that for the other nodes. For the surprise Kansei information, the changes were about $15-18 \%$, with the change at the corners of the mouth slightly higher than that for the other nodes.

In the next stage of our investigation, a result was counted as positive if the normalized polygon area change ratio increased and as negative if it decreased, after which the ratios of the corresponding test subjects were checked. These results are shown in Tables 2-4. As a general trend, it was found that, for positive Kansei, the change in $\Delta A$ for the inner end of the eyebrow and the upper parts of the eyes depended on the subjects, whereas that for the lower parts of the eyes always decreased, and that for the corners of the mouth always increased. For negative Kansei information, all normalized polygon area change ratios showed a decreasing trend, which was notably stronger at the inner end of the eyebrow and lower parts of the eyes. For surprise Kansei information, the $\Delta A$ values showed an increasing trend as a whole, which was stronger at the inner end of the eyebrow in particular.

Based on the above results, since the direction and amount of the change in $\Delta A$ depend on the individual, we believe a general trend can be speculated. However, it is thought that the Kansei information changes must be discerned individually. For discerning the three types of Kansei information from the results for the multiple test subjects in this study (positive Kansei information: minus at the lower parts of the eyes and plus at the corners of the mouth; negative Kansei information: minus at both the inner end of the eyebrow and lower part of the eyes; and surprise Kansei information: plus at both the inner end of the eyebrow and the corners of the mouth), it is thought that focusing on the directions and amounts is informative.

\section{Building and evaluation of Kansei discrimination algorithm}

Next, selecting a single test subject that had not participated in the earlier experiments, we examined the possibility of distinguishing each of the three targeted Kansei information types. It was decided to focus on a single test subject because, as previously mentioned, the directions and amounts of Kansei information change varies from individual to individual. Using this test subject, we then built an algorithm capable of identifying Kansei information from facial expressions. The average values and standard deviations of $\Delta A$ for the selected female test subject are shown in Table 5 (10 data points for each Kansei information type). The sizes of the changes for this subject were small overall.

Here, in order to determine which index is suitable from the four characteristic quantities, a distinction index formulated in a previous study (D.I., Sasaki et al. 2000) was calculated (Table 6). D.I. is a simplified index used when considering variations between categories (Eq. (4)).

$$
D . I .=\frac{\left|\mu_{\omega_{1}, x}-\mu_{\omega_{2}, x}\right|}{\sqrt{\sigma_{\omega_{1}, x}^{2}+\sigma_{\omega_{2}, x}^{2}}}
$$

Here, $\mu_{\omega_{1}, x}$ and $\sigma_{\omega_{1}, x}^{2}$ are the average and the variance for each category, respectively. In general, discrimination is easy when the D.I. is high. Reviewing the results shown in Table 6, it can be seen that the information about the corners of the mouth is most suitable for discriminating between positive and negative Kansei information, that the upper parts of the eyes are suitable for discriminating between the positive and surprise Kansei information, and that the corners of the mouth are suitable for discriminating between negative and surprise Kansei information.

In the next stage, we applied the holdout method (H-method) to determine error rates. The H-method is a cross-calibrated division learning technique used to determine error rates by interchanging the learning sample with the test sample. Using this method, the obtained samples were randomly divided into learning and test sample categories, which were then evaluated. This operation was repeated 1,000 times to calculate an average value, which was considered to be the error rate. The results of this process are shown in Table 7. These results showed that discrimination between positive and negative Kansei information and between negative and surprise Kansei information was

Table 5 Normalized polygon area change ratios* $(\%)$

\begin{tabular}{lccc}
\hline & $\begin{array}{c}\text { positive } \\
\text { Kansei }\end{array}$ & $\begin{array}{c}\text { negative } \\
\text { Kansei }\end{array}$ & $\begin{array}{c}\text { surprise } \\
\text { Kansei }\end{array}$ \\
\hline Inner end of the eyebrows & $0.05 \pm 2.47$ & $-18.80 \pm 2.70$ & $0.75 \pm 2.92$ \\
Upper part of the eyes & $6.07 \pm 4.27$ & $-15.08 \pm 6.65$ & $-0.42 \pm 3.75$ \\
Lower part of the eyes & $3.35 \pm 3.36$ & $-21.71 \pm 8.96$ & $2.28 \pm 3.57$ \\
Corners of the mouth & $2.69 \pm 1.34$ & $-10.81 \pm 1.03$ & $1.76 \pm 1.26$ \\
\hline
\end{tabular}

$*$ average \pm standard deviation

Table 6 D.I. compare of each polygon area

\begin{tabular}{ccccc}
\hline D.I. & $\begin{array}{c}\text { inner end } \\
\text { of the } \\
\text { eyebrow }\end{array}$ & $\begin{array}{c}\text { upper parts } \\
\text { of the eyes }\end{array}$ & $\begin{array}{c}\text { lower parts } \\
\text { of the eyes }\end{array}$ & $\begin{array}{c}\text { corners of } \\
\text { the mouth }\end{array}$ \\
\hline positive-negative & 5.15 & 2.68 & 2.62 & 8.00 \\
positive-surprise & 0.18 & 1.14 & 0.22 & 0.51 \\
negative-surprise & 4.91 & 1.92 & 2.49 & 7.72 \\
\hline
\end{tabular}


Table 7 H-method identification result compare

\begin{tabular}{cccc}
\hline Kansei & information & $\begin{array}{c}\text { The H-method } \\
\text { misconception } \\
\text { percentage* }(\%)\end{array}$ & $\begin{array}{c}\text { optimal } \\
\text { threshold }\end{array}$ \\
\hline positive-negative & $\begin{array}{c}\text { corners of the } \\
\text { mouth }\end{array}$ & $0.2 \pm 2.6$ & $-4.95 \%$ \\
positive-surprise & $\begin{array}{c}\text { upper parts of } \\
\text { the eyes }\end{array}$ & $21.1 \pm 13.5$ & $2.6 \%$ \\
negative-surprise & $\begin{array}{c}\text { corners of the } \\
\text { mouth }\end{array}$ & $0.0 \pm 0.0$ & $-5.2 \%$ \\
\hline
\end{tabular}

* average \pm standard deviation

possible at a rate of nearly $100 \%$ using the information obtained from the corners of the mouth. For positive and surprise Kansei information, an $80 \%$ discrimination rate could be achieved from the information of the upper parts of the eyes. The optimal threshold values for those discriminations were obtained statistically, and the three types of Kansei information for the target test subject could be identified using the algorithm discussed below.

In this system, it was first necessary to separately obtain learning data relating to facial expression Kansei information and to construct discrimination parameters and algorithms. Since the area where Kansei information tends to appear and the required identification precision vary between individual test subjects, this process will need to be automated in the future. Additionally, since we used general-purpose PCs equipped with built-in cameras in this study, we experienced a time lag of approximately two seconds when making determinations. In order to reduce this time lag, it will be necessary to consider using a computer and camera with higher specifications and avoid displaying the facial images and face nodes. Furthermore, while we computed the area change proportion of four normalized polygons in this study, it is highly likely that it will be possible to reduce the number of discrimination parameters for individual test subjects in the future, as well as to make real-time performance improvements by reducing the number of facial nodes and normalized polygon areas.

\section{Concluding Remarks}

In the first part of this study, a system that is capable of capturing and recognizing facial expressions using computer imagery was constructed. Based on an investigation involving multiple test subjects, the following results were obtained:

- For the three targeted Kansei information types, changes could be identified based on four specified normalized polygon area change ratios.

- For positive Kansei information, the changes at the corners of the mouth were noticeably higher than those at the other nodes. For negative Kansei information, the changes at the lower parts of the eyes were slightly higher than those at the other nodes. For surprise Kansei information, the changes at the corners of the mouth were slightly higher than those at the other nodes.

- Regarding the directions of the changes as a general trend, it was found that, for the positive Kansei information, the change in $\triangle A$ for the inner ends of the eyebrows and upper parts of the eyes depended on the test subjects, whereas that for the lower parts of the eyes always decreased, and that for the corners of the mouth always increased.

- For negative Kansei information, all $\Delta A$ measurements showed a decreasing trend, which was notably stronger at the inner ends of the eyebrows and lower parts of the eyes.

- For surprise Kansei information, $\Delta A$ measurements showed an increasing trend as a whole, which was notably stronger at the inner ends of the eyebrows.

In the second part of this study, we explored the responses of a single test subject to determine the relationship between facial expressions and Kansei information, and then formulated an algorithm based on the results. A single test subject was used in this phase because the directions and amounts of the change related to Kansei information vary from individual to individual.

- When discriminating between positive and negative Kansei information, and between negative and surprise Kansei information, discrimination rates near $100 \%$ were found to be possible from the information obtained from the corners of the mouth.

- For discrimination between positive and surprise Kansei information, a discrimination rate of approximately $80 \%$ was found possible using information obtained from the upper parts of the eyes.

Thus, although it is currently necessary to prepare an individual template image for the seven points of each test subject in advance and to adjust the parameters after measuring the Kanseirelated facial expressions, it is clear that inexpensive and near real-time computer vision systems capable of extracting facial expression Kansei information can be built. We believe that such systems have potential applications in automatic evaluation and design through decision support using Kansei information. Our future challenges include reduction of the amount of processing required for creating template images, as well as that for other processes, and the construction of a real-time Kansei-related facial information recognition system. 


\section{Acknowledgment}

Part of this work was supported by Grant-in-Aid for Scientific Research (KAKENHI A, 23248043).

\section{References}

Asaumi, H., H. Nishina and Y. Hashimoto (1995) Studies on amenity of indoor plants, Acta Horticulturae, 391: 111-118.

Ayinde, O. and Y.-H. Yang (2002) Face recognition approach based on rank correlation of Gabor-filterd images, Pattern Recognition, 35: $1275-1289$.

Fujimura T. and N. Suzuki (2008) The effects of temporal information and configuration changes on the recognition of emotional facial expressions: From a dimensional perspective, The Japanese Journal of Cognitive Psychology, 5(1): 53-61.

Mehirabian, A. (1986) "Silent messages: Implicit communication of emotions and attitudes", SEIBUNSHINSHA Publishing Co., Ltd, $255 \mathrm{pp}$.
Sasaki, Y., M. Suzuki, P. Chen and T. Saito (2000) The Development of Intelligent Green Soybean Selector by Machine Vision Possibility of Class Selecting Used by Form Information, Journal of the Japanese Society of Agricultural Machinery, 62(6): 92-97.

Sasaki, Y., K. Tajima and M. Nakazawa (2007) Examination of Kansei Inference Using Facial Expression Information by Tracking Facial Features, Agricultural Information Research, 16(4): 205-211.

Sasaki, Y., N. Arakawa, Y. Sato, H. Yajima and K. Ito (2009) Development and Evaluation of 3-D Facial Expression-Kansei Estimation System, Agricultural Information Research, 18(1): 1723.

Sasaki, Y. (2011) Detection of the Intention from Gestures of Workers for a Kansei Agri-Robot, Agricultural Information research, 20(1): $13-18$.

Zisheng L., J. Imai and M. Kaneko (2010) Facial Expression Recognition Using Facial-component-based Bag of Words and PHOG Descriptors, Information and Media Technologies, 64(2): 230-236.

Received April 24, 2013

Accepted March 31, 2014

Agro-informatics \& Technology 